\title{
Comparison of the Effects and Mechanism of the Curcumin with Different Drugs in Experimental Vasospasm After Subarachnoid Hemorrhage
}

\author{
Hasan Emre AYDIN¹, Nuriye Ezgi BEKTUR², Zuhtu OZBEK², Setenay ONER ${ }^{4}$, Cengiz BAYCU², \\ Fatma Sultan KILIC ${ }^{5}$ \\ 1'Dumlupınar University, Department of Neurosurgery, Kutahya, Turkey \\ ${ }^{2}$ Osmangazi University, Department of Histology, Eskişehir, Turkey \\ ${ }^{3}$ Osmangazi University, Department of Neurosurgery, Eskisehir, Turkey \\ ${ }^{4}$ Osmangazi University, Department of Biostatistics, Eskisehir, Turkey \\ ${ }^{5}$ Osmangazi University, Department of Pharmacology, Eskisehir, Turkey
}

\section{ABSTRACT}

AIM: Cerebral vasospasm following subarachnoid hemorrhage (SAH) is the most important complication that effects the mortality and morbidity of patients with intracranial aneurysm. Today, the mechanisms of vasospasm are not understood in spite of experimental and clinical researches. The aim of our study was to investigate the effects of curcumin on vasospasm following SAH.

MATERIAL and METHODS: In this study, 64 rats (200-250 g weight) were divided into 7 groups. Group 1: having no treatment after $\mathrm{SAH}$; Group 2: treatment with nimodipine after SAH; Group 3: treatment with nicorandil after SAH; Group 4: treatment with sildenafil citrate after SAH; Group 5: treatment with $150 \mathrm{mg} / \mathrm{kg}$ curcumin after SAH; Group 6: treatment with $300 \mathrm{mg} / \mathrm{kg}$ curcumin after SAH, Group 7: treatment with $600 \mathrm{mg} / \mathrm{kg}$ curcumin after SAH. The experimental SAH was induced by injection of autologous blood into the cisterna magna. After medical treatment, in the first hour, blood was taken for quantified the levels of TNF-a, IL-1 $1 \beta$ and IL-6. Then, cerebrum and cerebellum were removed for analysis. Basilar artery luminal diameter was measured and apoptotic cell count was performed with tissue samples.

RESULTS: Histopathological findings showed that, in sufficient dose, curcumin dilated the basilar artery beside anti-oxidant effect. CONCLUSION: Curcumin can be used for the treatment of vasospasm as a new medical drug.

KEYWORDS: Curcumin, Nicorandil, Nimodipine, Sildenafil citrate, Subarachnoid hemorrhage

\section{INTRODUCTION}

S ubarachnoid Hemorrhage (SAH) is an acute-onset, usually arterial or rarely venous bleeding into cerebral subarachnoid space or between the arachnoid and pia mater (19). It is commonly seen after head trauma, while $75-80 \%$ of the spontaneous SAH results from the rupture of intracranial aneurysms (11). Despite using the available treatments, the mortality is high. Although there are numerous experimental and clinical studies, the exact underlying mechanisms for the associated pathological conditions such as vasospasm remain to be identified. Major histological change during the cerebral vasospasm after SAH is the endothelial cell injury induced by apoptosis.

Nimodipine is a dihydropidin-group calcium channel blocker that is selective to the cerebral vessels. It is the only used in clinical practice in patients diagnosed as SAH $(3,24,26)$. 
Because of its high selectivity, low-dose nimodipine results in dilatation of cerebral vessels and prevents cerebral vasospasm without leading to a prominent decrease in systemic blood pressure $(7,21)$.

Nicorandil is a drug leading to the opening of adenosine triphosphate (ATP)-dependent potassium channels and to the release of nitric oxide (NO). Although it is commonly used for the treatment of angina pectoris and acute heart failure, current treatment approaches also include use of the drug in ischemia-reperfusion injury. The drug improves the chronic vasospasm and vascular contraction induced by subarachnoid hemorrhage $(12,22)$.

Sildenafil is a peripheral vasodilator agent inhibiting the phosphodiesterase type $V$ enzyme selectively. The drug is used for angina pectoris, erectile dysfunction, pulmonary hypertension, Reynaud phenomenon, and vertebrobasilar insufficiency $(5,15)$. Because the drug increases the blood flow, it prevents neuronal injury induced by vasospasm following SAH (15).

Curcumin was isolated in 1842 by Vogel and Pelletier from the roots of turmeric, which is yellow-flowered plant known for nearly a thousand years. Up to the present day, approximately 4000 studies have found that curcumin exerts antibacterial, anti-inflammatory, hypoglycemic, anti-oxidant and antifungal effects. It is also well known that curcumin results in sympathetic blockage and NO release, leading to decreased blood pressure through vasodilatation $(1,8,13)$.

The aim of this study was to examine the effects of curcumin, in addition to its well-known vasodilator effects also used for disorders in locations other than the central nervous system (CNS), on vasoconstriction induced by ischemia and various factors and on apoptosis induced by the blood-brain barrier dysfunction following brain injury, as well as on the prevention of activation of inflammatory pathways (28).

\section{MATERIAL and METHODS}

The experimental part of this study was conducted at the Medical and Surgical Experimental Research Center (MESERC), Laboratory of Experimental Animals. The histological preparations, basilar artery diameter measurement and histopathological evaluation were performed at the Laboratory of Histology and Embryology, and immunochemical studies were carried out at the Laboratory of Pharmacology of Osmangazi University, Eskisehir, Turkey. The study protocol was approved by the Osmangazi University Ethics Committee for the Experimental Use of Animals (Date: 27.02.2013, Number: 55-318).

A total of 64 female Sprague-Dawley rats weighing 200-250 $g$ were used:

Group 1: Untreated group with SAH

Group 2: Nimodipine-treated SAH group

Group 3: Nicorandil-treated SAH group

Group 4: Sildenafil-treated SAH group

Group 5: $150 \mathrm{mg} / \mathrm{kg}$ of curcumin-treated SAH group
Group 6: $300 \mathrm{mg} / \mathrm{kg}$ of curcumin-treated SAH group

Group 7: $600 \mathrm{mg} / \mathrm{kg}$ of curcumin-treated SAH group

In animals in Group 1-7, under general anesthesia, $0.1 \mathrm{~mL}$ of nonheparinized autologous arterial blood was withdrawn from the heart and given into the subarachnoid space via puncture with a $3 G$ cannula at the occipito-atlantal distance. Powdered drugs (Nimodipine, nicorandil, sildenafil and curcumin) were dissolved mechanically in the mixture of $0.06 \mathrm{mg} / 1 \mathrm{mg}$ saline/DMSO. In animals with SAH, a single dose of $0.05 \mathrm{mg}$ nimodipine, $10 \mathrm{mg} / \mathrm{kg}$ nicorandil, $5 \mathrm{mg} / \mathrm{kg}$ sildenafil, or 150 , 300 or $600 \mathrm{mg} / \mathrm{kg}$ curcumin was injected intraperitoneally. The animals in Group 1 were treated with a single intraperitoneal dose of $0.06 \mathrm{mg} / 1 \mathrm{mg}$ saline/DMSO after inducing SAH. In the first hour of the treatment, $2 \mathrm{~mL}$ of arterial blood was drawn for the measurements of IL-1 $\beta$, IL- 6 and TNF- $a$ by the ELISA method (eBioscience, Bender MedSystems, Vienna, Austria).

Then, under general anesthesia, bilateral fronto-parietooccipital craniectomy was performed. The cerebrum, cerebellum and brain stem above the foramen magnum were totally removed while keeping the anatomic integrity. Macroscopically, all animals with SAH demonstrated diffuse subarachnoid hemorrhage in the basal surface of brain stem and around of the vertebral and basilar arteries. The terminal deoxynucleotidyITransferase Biotin - dUTP Nick End Labeling (TUNEL) method was used to determine whether apoptotic cells were present in the transverse sectioned tissue samples from the brain, brain stem and basilar artery. A blinded observer evaluated the number of apoptotic cells on four TUNEL-stained tissue sections from each animal by using a computer-assisted microscope Olympus BX 51 (Japanese) and the BAB Bs200pro software. The diameter of the basilar artery was determined by using a microscope and the computer software. A total of ten measurements were performed on the vessel lumen in four-sections from each animal.

\section{Statistical Analysis}

Statistical analysis was performed by using the SigmaStat 3.5 and IBM SPSS Statistics 21 package software. Descriptive data were expressed as median $25-75 \%$. Because data were not distributed normally, the data on the number of apoptotic cells, the diameter of basilar artery and the measurements of interleukin 1-Beta (IL-1 $\beta$ ), IL-6 and tumor necrosis factoralpha (TNF-a) were analyzed by using Kruskal-Wallis One-way Analysis. The multiple comparison test used for cytokine levels and basilar artery diameter was Dunn's method and that for the number of apoptotic cells was the Tukey test. Statistical significance was set as $p<0.001$.

\section{RESULTS}

\section{Histological Findings}

The tissue sections for microscopic analysis were obtained from the brain stem and around the basilar artery. The examination under the light microscope revealed a healthy basilar artery structure, neuronal body and neuroglia cells in the control group. TUNEL staining showed that the DNA 
fragments in apoptotic cells were negative in the brain tissue of the control animals (Figure 1).

Animals with untreated SAH demonstrated marked undulation in lamina elastica interna and distortion in the endothelium resulting from the vasoconstriction of the basilar artery. There was twisting in neuronal body extensions as well as shrinkage and contraction in nerve support cells and neurons during the apoptotic process. In addition, a large number of neuroglia cells and neuron bodies were positive for TUNEL reaction in this group (Figure 2).

In the $\mathrm{SAH}+$ Nimodipine group, although some regions of the lamina elastica interna of the basilar artery showed undulation, there was a dilatation of the artery lumen in general. There was also reduction, shrinkage and eosinophilic cytoplasm in neuron bodies and neuroglia cells. TUNEL-positive cells were also seen with being more less severe compared to the SAH group (Figure 3).
In the SAH+Nicorandil group, dilatation of basilar artery, decreased undulation in lamina elastica interna, reduction and shrinkage in neuron bodies, and TUNEL-positive cells were present (Figure 4).

On the other hand, Sildenafil-treated animals with SAH not only showed dilated basilar artery but also had undulation in lamina elastica interna. Although some neuron bodies had pyknotic nuclei that were pushed to a cell pole and became necrotic, there were also healthy neuron bodies and extensions. In this group of animals, TUNEL-positive cells were mostly found in neuroglia cells (Figure 5).

In the $\mathrm{SAH}+$ Curcumin 150 group, the undulation in lamina elastica interna was decreased in parallel to the dilatation in the lumen of basilar artery. With a great proportion of neuron bodies and neuroglia cells being healthy, the number of TUNEL-positive cells was lower than in the SAH group (Figure 6)

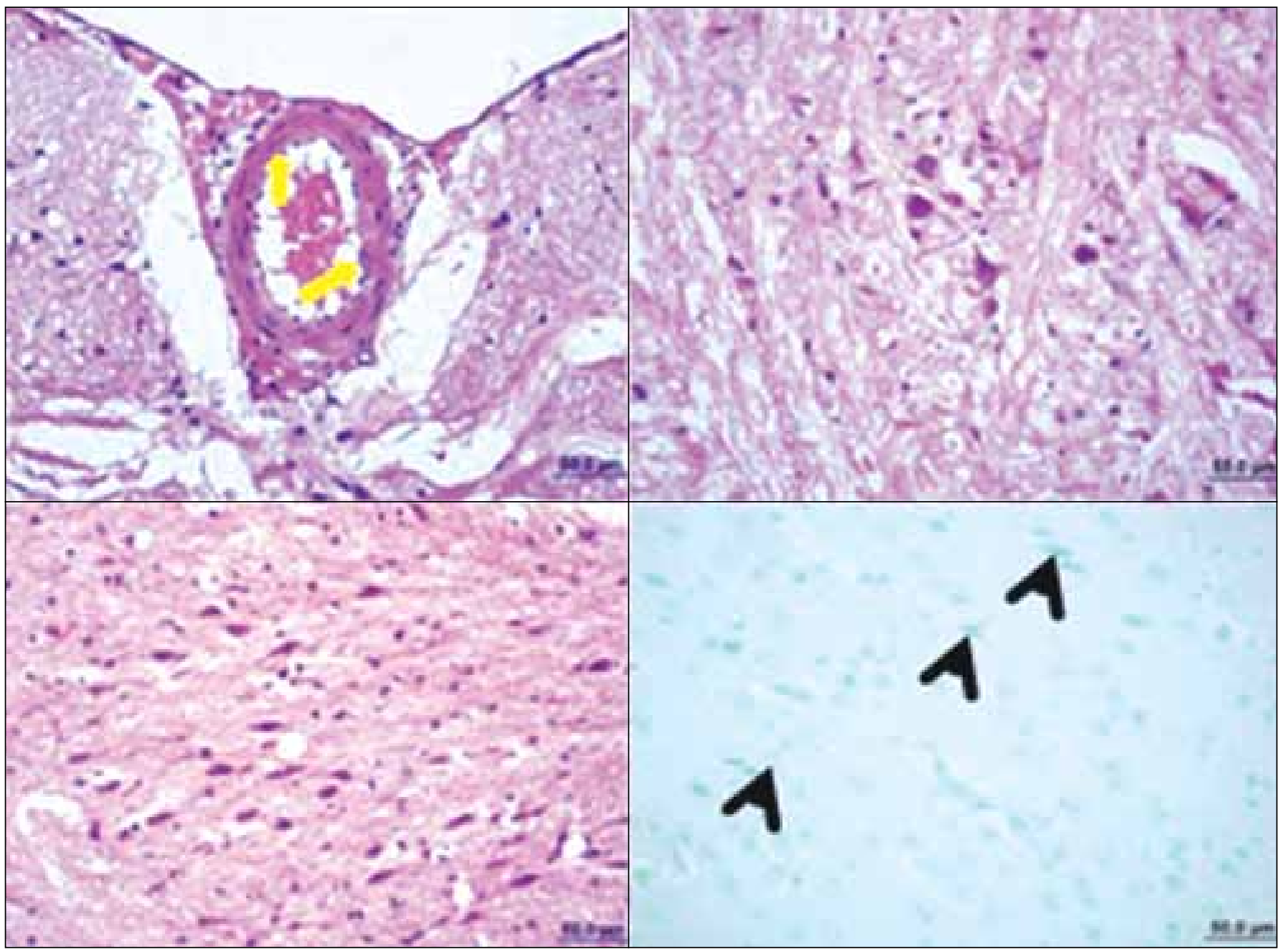

Figure 1: The tissue sections for microscopic analysis were obtained from the brain stem and around the basilar artery. The examination under the light microscope revealed a healthy basilar artery structure, neuronal body and neuroglia cells in the control group. TUNEL method staining showed that the DNA fragments in apoptotic cells were negative in the brain tissue of the control animals. 

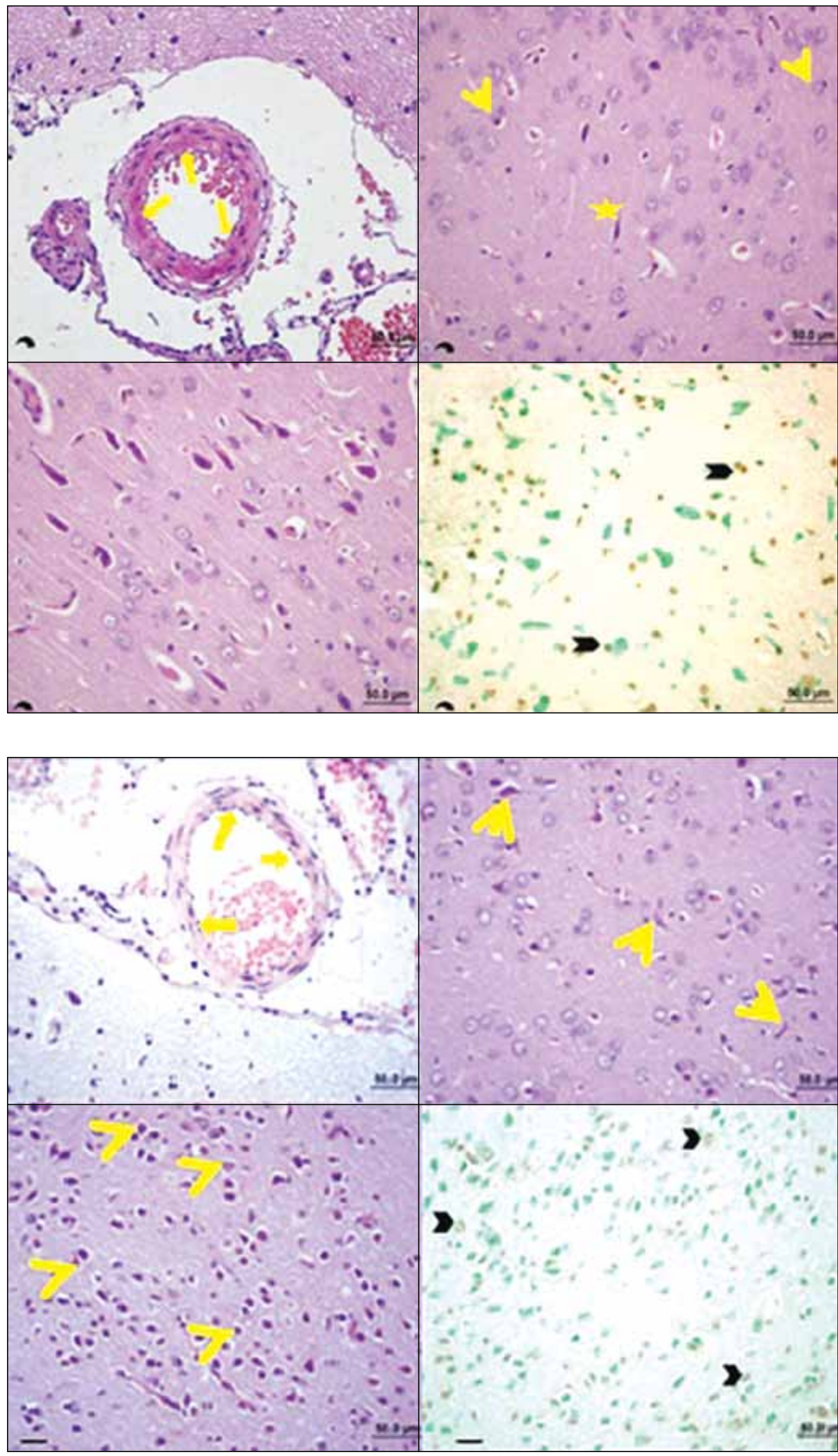

Figure 2: Animals with untreated SAH demonstrated marked undulation in lamina elastica interna and distortion in the endothelium resulting from the vasoconstriction of basilar artery. There was twisting in neuronal body extensions as well as shrinkage and contraction in nerve support cells and neurons during the apoptotic process. In addition, a large number of neuroglia cells and neuron bodies were positive for TUNEL reaction in this group.
Figure 3: In the SAH+Nimodipine group, although some regions of the lamina elastica interna of the basilar artery showed undulation, there was dilatation of the artery lumen in general. There was also reduction, shrinkage and eosinophilic cytoplasm in neuron bodies and neuroglia cells.

TUNEL-positive cells were also seen with being more less severe compared to the SAH group. 
In the $\mathrm{SAH}+$ Curcumin 300 group, the dilatation in the basilar artery increased and the undulation of lamina elastica interna disappeared. Nissl granules and neuronal body extensions were healthy and clear, and the number of TUNEL-positive cells was lower compared to the SAH group (Figure 7). However, there was no difference between the groups treated with Curcumin 150 and $300 \mathrm{mg}$.

In the $\mathrm{SAH}+$ Curcumin 600 group, there was no undulation in the lamina elastica interna due to the increased dilatation in the lumen of the basilar artery compared to all other experimental groups. The cells in this group showed less positive reaction to TUNEL staining (Figure 8). However, there was no significant difference.

\section{Cytokine Levels}

After the SAH, there was no difference in IL-6 levels $(p>0.05$ ) but the levels of TNF- $\alpha$ and IL- $1 \beta$ differed significantly between the groups $(p<0.001)$ (Figures $9-11)$. Dunn's method was used to compare the groups and a significant difference was found between the groups treated with nimodipine, nicorandil, sildenafil, and $150 \mathrm{mg} / \mathrm{kg}, 300 \mathrm{mg} / \mathrm{kg}$ and $600 \mathrm{mg} /$ $\mathrm{kg}$ curcumin $(\mathrm{p}<0.001)$

\section{Apoptotic Cell Count and the Diameter of the Basilar Artery}

The diameter of the basilar artery did not show a normal distribution pattern and the Kruskal-Wallis One-way analysis showed a statistically significant difference $(p<0.001)$. The intergroup differences were analyzed by using Dunn's method and a significant difference was found between SAH-group and the groups treated with nimodipine, nicorandil, sildenafil and 300 and $600 \mathrm{mg} / \mathrm{kg}$ of curcumin $(\mathrm{p}<0.001)$ (Figure 12). Importantly, the effect of curcumin on vessel diameter was not dose-dependent and the significant effect was obtained with $300 \mathrm{mg} / \mathrm{kg}$ and $600 \mathrm{mg} / \mathrm{kg}$ of curcumin.

Because the number of apoptotic cells did not show a normal distribution, the Kruskal Wallis One-Way analysis was used and a statistical significant difference was found $(p<0.001)$. The intergroup difference was analyzed by using Tukey test. The number of apoptotic cells decreased significantly in the $\mathrm{SAH}+$ Curcumin $300 \mathrm{mg}$ group compared to the untreated SAH group $(p<0.001)$. Similarly, the Curcumin $300 \mathrm{mg}$ group had a decreased number of apoptotic cells compared to the nimodipine and nicorandil groups, and sildenafil had no effect on apoptosis (Figure 13).

\section{DISCUSSION}

Vasospasm is a complicated problem seen after subarachnoid hemorrhage and the underlying mechanism is not clear. The increased blood in the subarachnoid space is known to initiate the inflammatory process, which results in increased

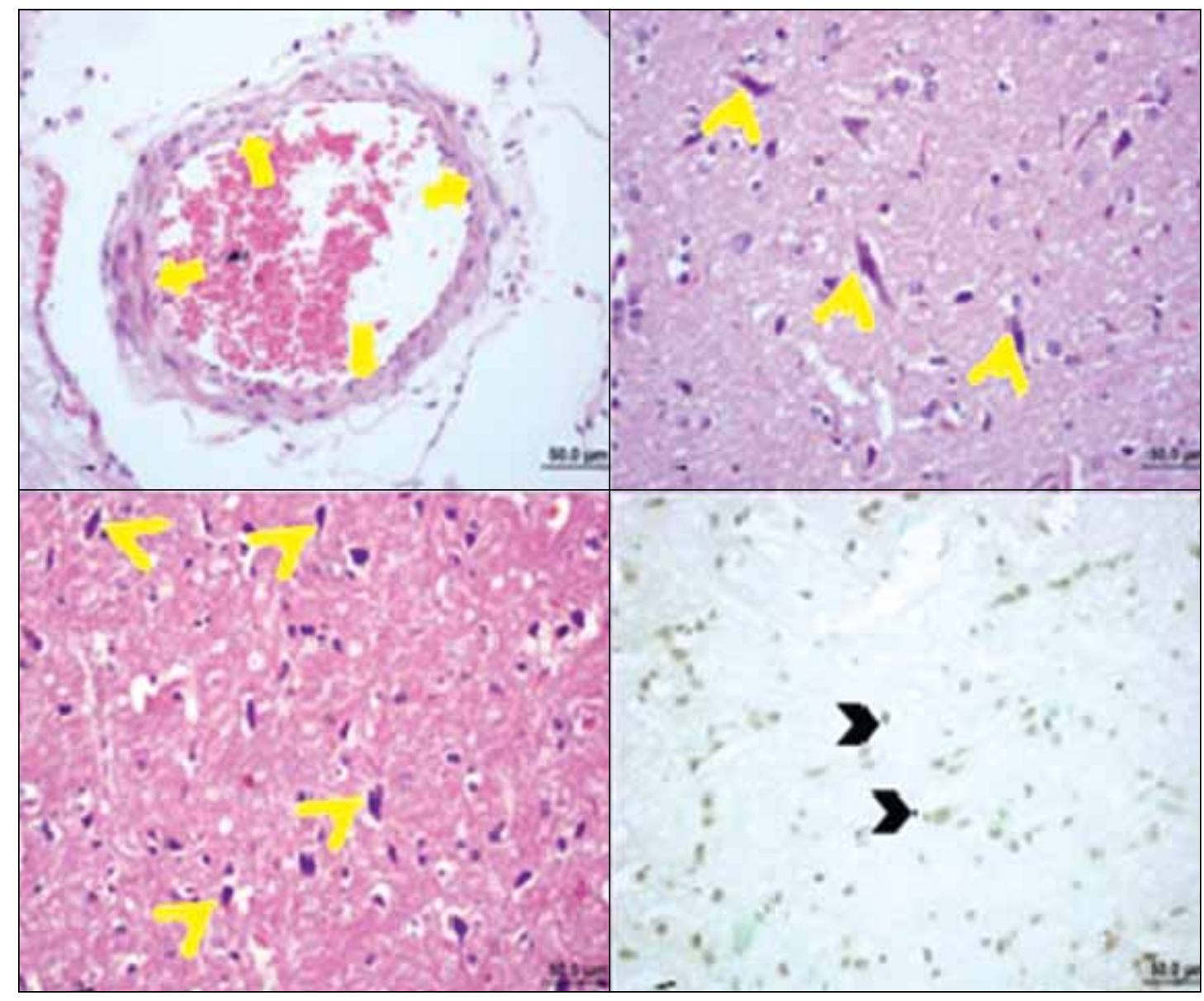

Figure 4: In the $\mathrm{SAH}+$ Nicorandil group, dilatation of basilar artery, decreased undulation in lamina elastica interna, reduction and shrinkage in neuron bodies, and TUNELpositive cells were present. 
cytokine levels, increased apoptosis and decreased diameter of vessel lumen, leading to the vasospasm. Until recent years, numerous hypotheses have been developed on this issue and various experimental animal models (rat, rabbit and dog) have been used to test different drugs. The experimental rat model induced by injection of non-heparinized autologous blood into the cisterna magna has been suggested to be applicable and reliable as it resembles the clinical findings well. Therefore, this rat model is the most commonly used experimental method in neuropharmacological studies on the prevention of vasospasm.
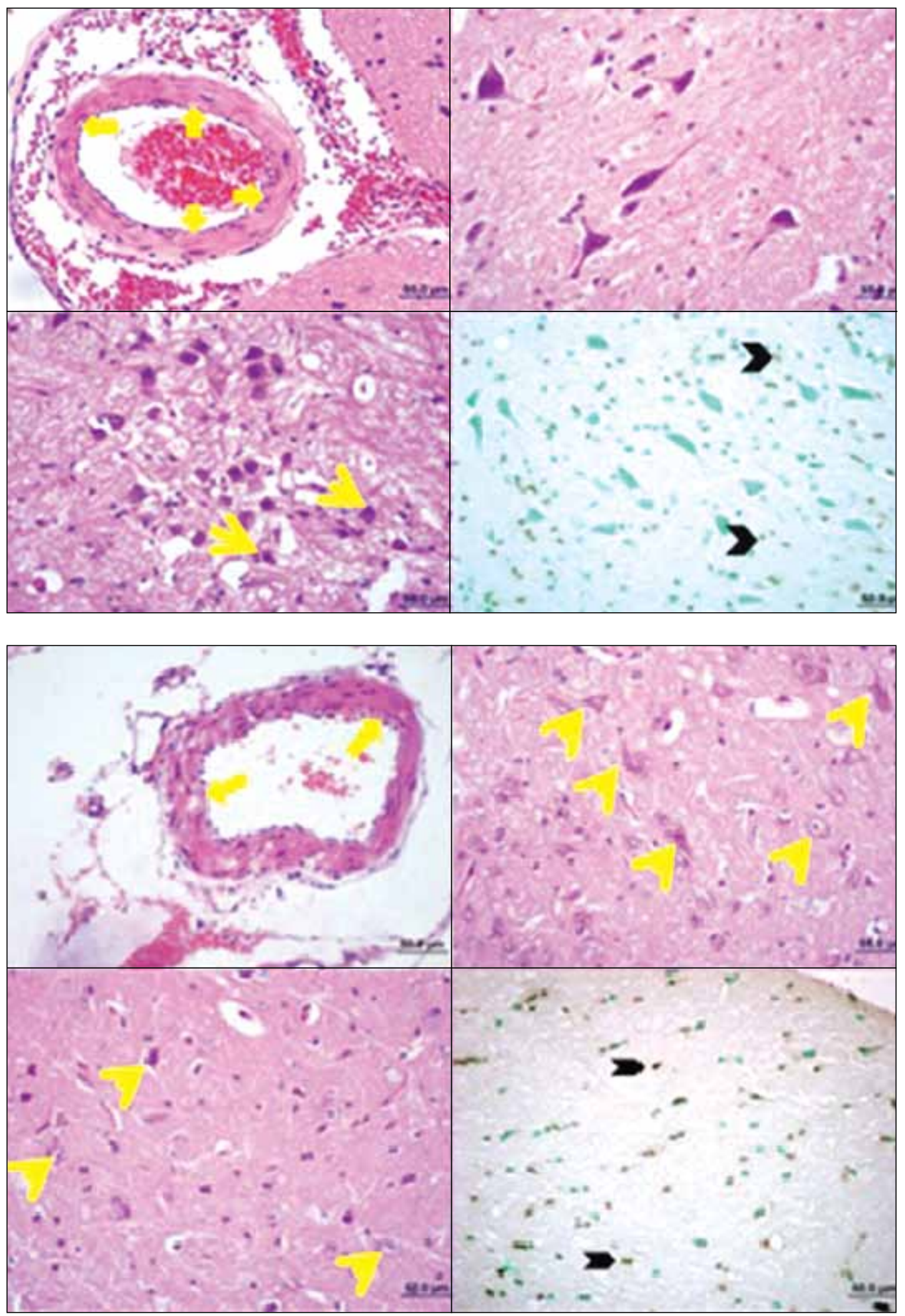

Figure 5: On the other hand, Sildenafil-treated animals with SAH not only showed dilated basilar artery but also had undulation in lamina elastica interna. Although some neuron bodies had pyknotic nuclei that was pushed to a cell pole and became necrotic, there were also healthy neuron bodies and extensions. In this group of animals, TUNEL-positive cells were mostly found in neuroglia cells.
Figure 6: In the

$\mathrm{SAH}+$ Curcumin 150 group, in parallel to the dilatation in the lumen of basilar artery, the undulation in lamina elastica interna was decreased. With a great proportion of neuron bodies and neuroglia cells being healthy, the number of TUNEL-positive cells was lower than SAH group. 

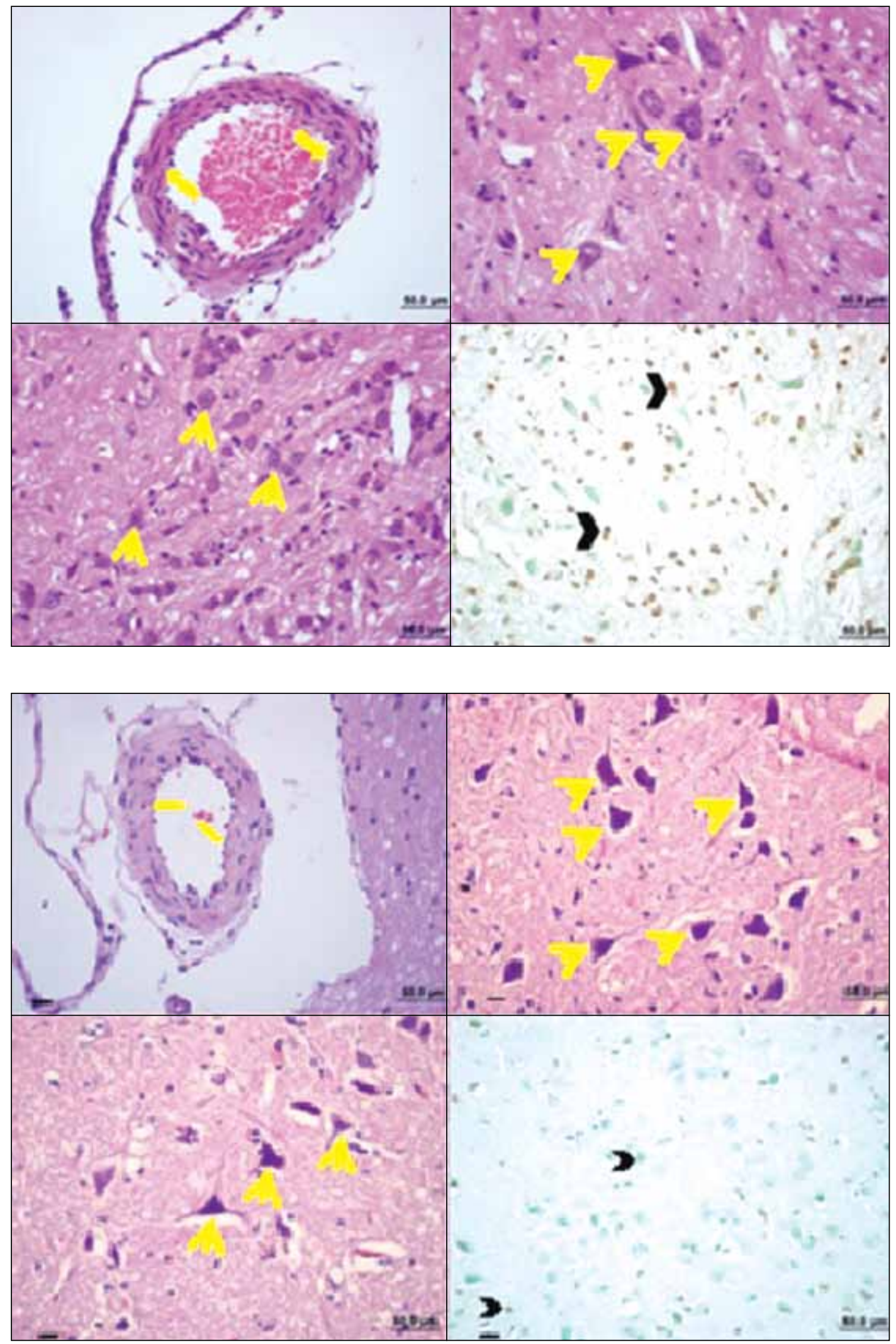

Figure 7: In the

$\mathrm{SAH}+$ Curcumin 300 group, the dilatation in basilar artery was increased and the undulation of lamina elastica interna disappeared. Nissl granules and neuronal body extensions were healthy and clear and the number of TUNELpositive cells was lower compared to the SAH group.
Figure 8: In the $\mathrm{SAH}+$ Curcumin 600 group, there was no undulation in lamina elastica interna due to the increased dilatation in the lumen of basilar artery compared to all other experimental groups, the cells in this groups showed less positive reaction to TUNEL staining. 


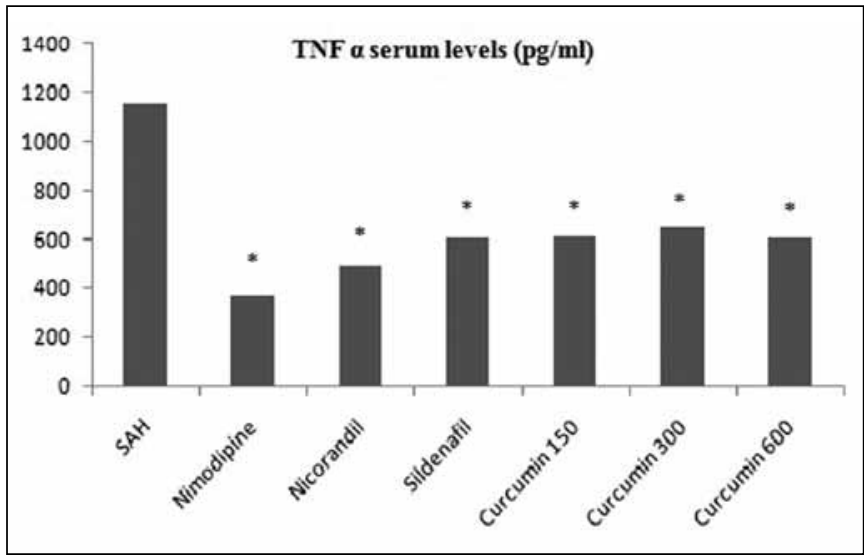

Figure 9: Serum levels of IL-6 (pg/ml).

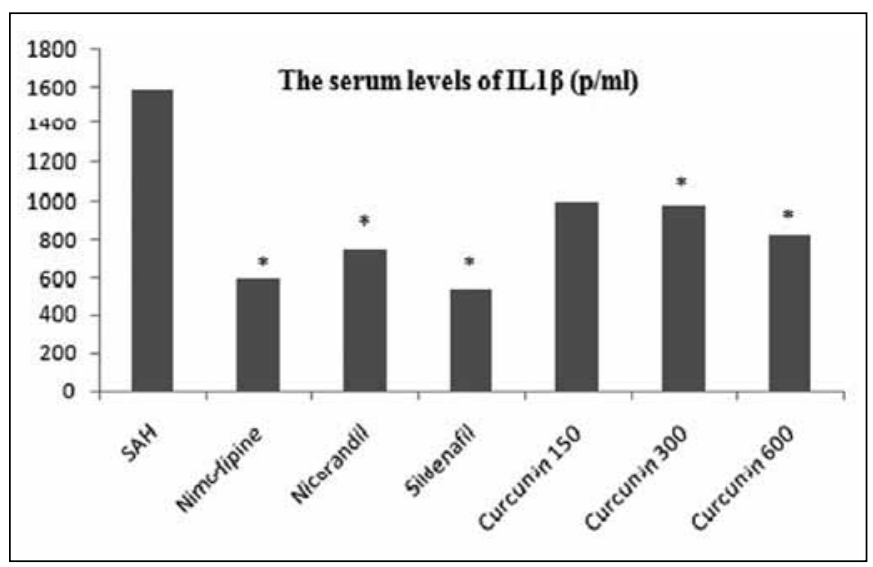

Figure 11: Serum levels of IL-1 $\beta(\mathrm{pg} / \mathrm{ml})$.

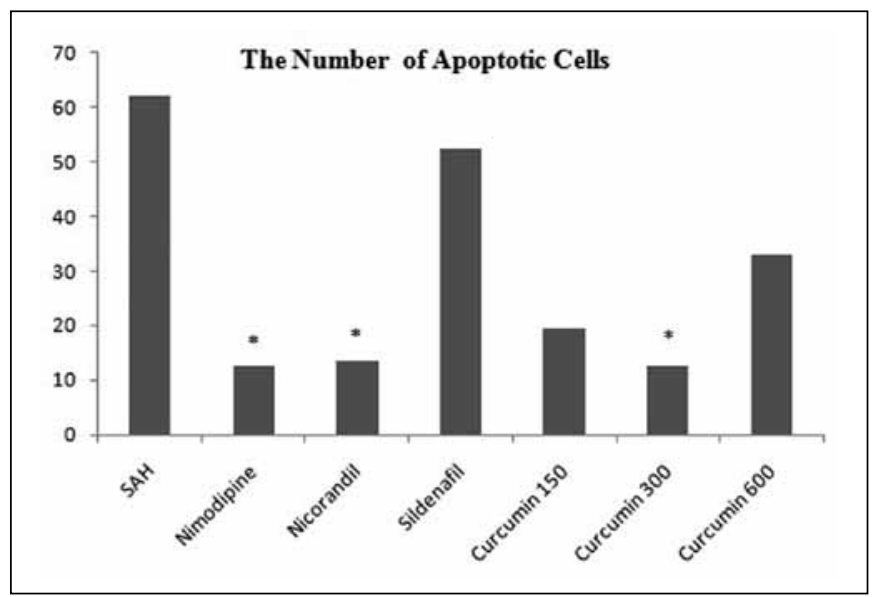

Figure 13: The number of apoptotic cells shown graphically. The significance between the groups is indicated with the marker.

Nimodipine is an effective and safe treatment for symptomatic vasospasm after SAH $(6,20)$. Regional administration of the drug has a potent vasodilatory effect even in low doses. Because high doses of nimodipine have been shown in dose-dependent experimental studies to result in no increase

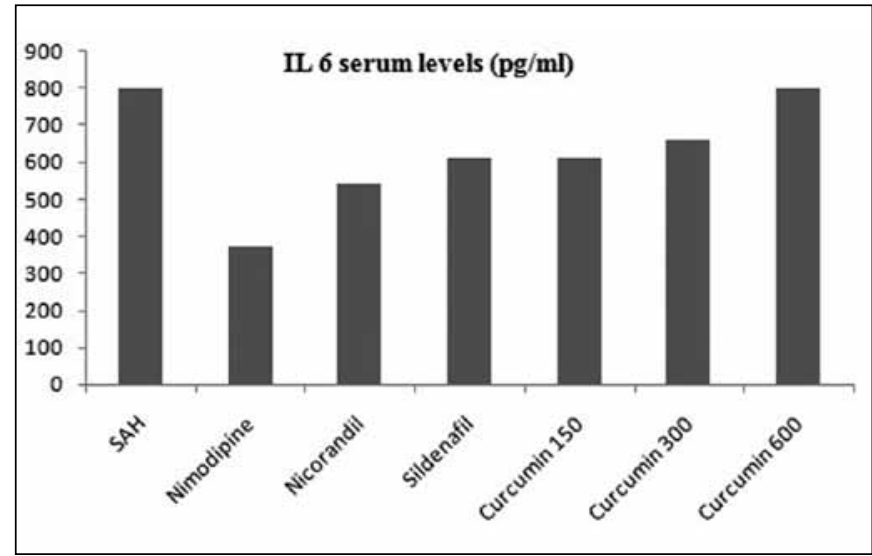

Figure 10: Serum levels of TNF-a (pg/ml).

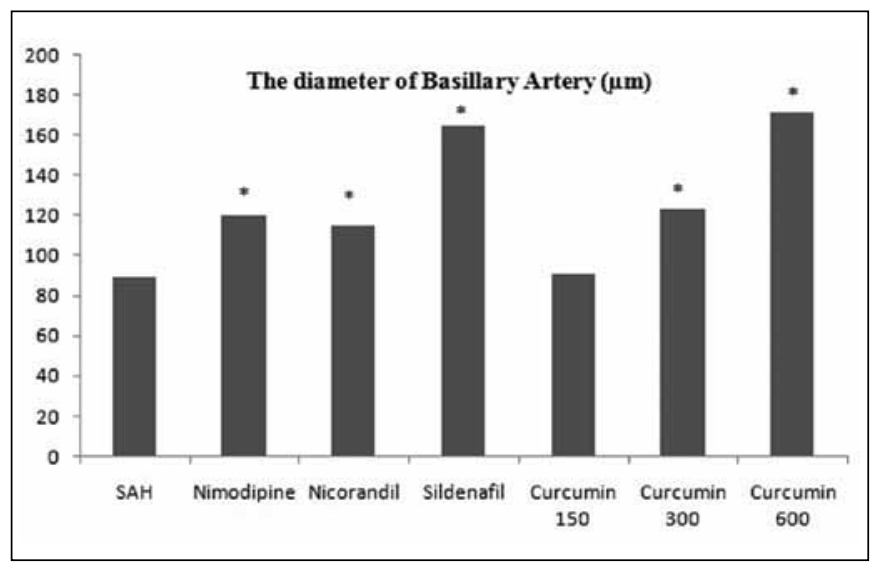

Figure 12: The diameter of the basilar artery is shown graphically. The significance between the groups is indicated with the marker.

in dilatation, high doses of nimodipine should be avoided in order to prevent the hypotension-induced decrease in cerebral perfusion pressure (21). Moreover, prophylactic use of nimodipine may prevent the secondary injury resulting from the late cerebral infarction. The American Stroke Association has recommended immediate oral nimodipine treatment for all patients in the intensive care unit (26). Accordingly, in the present study, apoptotic cell number was decreased and vasodilatation was increased in nimodipine-treated animals compared to the untreated animals with SAH $(p<0.001)$. Nimodipine decreased the TNF- $\alpha$ and IL-1 $\beta$ levels, with no effect on IL-6 level.

Previous studies have shown that sildenafil citrate has no effect on apoptosis, but leads to marked dilatation in spastic cerebral vessels $(2,18,23)$. Sildenafil has been also suggested to increase the angiogenesis and the intracerebral blood flow selectively following ischemic stroke (5). In the present study, in parallel with the literature, sildenafil had a more potent vasodilatory effect than nimodipine with no effect on apoptosis. Thus, in addition to nimodipine -for which effectiveness has been established- sildenafil may be considered for use in cerebral SAH treatment due to the potent vasodilatory effect of the drug. 
The relaxant effect of nicorandil on brain vessels is dosedependent and increasing doses lead to hyperpolarisationinduced dilatation. High-dose nicorandil $(20 \mu \mathrm{g} / \mathrm{kg} / \mathrm{min})$ has been shown to result in decreased cerebral vasoconstriction in angiography $(12,14)$. In the present study, nicorandil also led to decreased number of apoptotic cells and increased vasodilatation $(p<0.001)$. Nicorandil is a commonly used drug that opens the ATP-dependent potassium channels and leads to NO release and has been shown to induce vasodilatation not only in the heart but also in the cerebral vessels.

Curcumin administered intraperitoneally at a dose of 30-300 $\mathrm{mg} / \mathrm{kg}$ has been found to be effective in brain ischemiareperfusion studies (27). Considering the fact that treatment is initiated in the emergency department as soon as possible after making the definite $\mathrm{SAH}$ diagnosis, curcumin was used intraperitoneally at 3 different doses $(150,300$ and $600 \mathrm{mg} /$ $\mathrm{kg}$ ) in an experimental SAH model. Compared to the SAH group, animals treated with $300 \mathrm{mg} / \mathrm{kg}$ of curcumin showed significant differences in apoptosis and vessel diameter $(p<0.001)$. Although this effect increased with increasing doses of curcumin, there was no statistical difference. After analyzing all the parameters, the most effective dose of curcumin was determined to be $300 \mathrm{mg} / \mathrm{kg}$. Moreover, in the animal groups treated with curcumin -which prevents the inflammatory response by removing the clots- showed decreased IL-1 $\beta$ and TNF-a levels but unchanged IL- 6 levels compared to the untreated animals with SAH. Accordingly, previous studies have shown that curcumin also decreases the IL-6 release and thus, decreases the brain edema leading to the secondary neuronal injury after SAH $(17,25)$. Curcumin at a dose of 300 $\mathrm{mg} / \mathrm{kg}$ was as effective as nimodipine, nicorandil and sildenafil in dilating the vessel.

Interleukins and TNF- $\alpha$ are the acute phase reactants released in response to the inflammatory process induced by the blood filling into the subarachnoid space (9). IL-1 $1 \beta$, TNF- $\alpha$ and IL10 reach the peak level within 12 hours in response to the inflammatory process (3). Fassbender et al. (11) have found that release of IL- $1 \beta$, IL- 6 and TNF- $\alpha$ is a characteristic finding after SAH. On the other hand, another study has reported increased IL- 6 levels but unchanged TNF-a level after SAH $(16,23)$. Previous studies on the cytokine levels following $\mathrm{SAH}$ and vasospasm have reported inconsistent results. Particularly, the measurements in CSF and blood -even if simultaneous- have not been found to be parallel. The major finding in these studies was increased cytokine levels due to the neurological deterioration associated with the blood in the subarachnoid space (10). In the present study, curcumin led to a decrease in cytokine levels -particularly in IL-1 $\beta$ and TNF- $a$ levels- and decreased the inflammatory response, in parallel to the literature. Sildenafil has been also reported to decrease the cytokine level in the acute period following SAH $(2,4,13)$.

\section{- CONCLUSION}

Curcumin, which is an antioxidant substance and has been used in a large number of studies, was found to induce vasodilatation in cerebral arteries when used at an appropriate dose. Additionally, a peripheral vasodilatory agent -Sildenafiland the potassium channel opener agent "Nicorandil" were also found to result in cerebral vasodilatation, suggesting that these drugs may be also used in the treatment of vasospasm as an alternative to nimodipine. Further studies on the medical treatment of vasospasm, for which the underlying mechanism is not clear, are needed.

\section{- REFERENCES}

1. Akpolat M, Tarladacalısır YT, Uz HY, Metin MS, Kızılay G: Effects of curcumin in the medical treatment of cancer. New Med J 27: 142-147, 2010

2. Altinors N, Celasun B, Ozen O, Cekinmez M, Caner H, Atalay B: Systemic administration of phosphodiesterase $V$ inhibitor, Sildenafil Citrate, for attenuation of cerebral vasospasm after experimental subarachnoid hemorrhage. Neurosurgery 59(5):1102-1108, 2006

3. Athar $\mathrm{KM}$, Levine $\mathrm{MJ}$ : Treatment options for cerebral vasospasm in aneurysmal subarachnoid hemorrhage. Neurotherapeutics 9: 37-43, 2012

4. Bombarda G, Sabino JPJ, Silva C, Fazan R, Salgado CM, Salgado HC: Role of cGMP and cAMP in the hemodynamic response to intrathecal sildenafil administration Clinics 66(8): 1407-1411, 2011

5. Bozgeyik Z, Berilgen S, Ozdemir H, Tekatas A, Ogur, E: Evaluation of the effects of Sildenafil on vertebral artery blood flow in patients with vertebro-basilar insufficiency. Korean $\mathrm{J}$ Radiol 9: 477-480, 2008

6. Cho W, Kang H, Kim JE, Kwon O, Oh CW, Son YJ, Kwon BJ: Effect of intra-arterial nimodipine infusion for the treatment of symptomatic cerebral vasospasm following an aneurysmal subarachnoid hemorrhage. Interv Neuroradiol 17: 169-178, 2011

7. Cook AD: Mechanisms of cerebral vasospasm in subarachnoid haemorrhage. Pharmacol Ther 66: 259-284, 1995

8. Dewar AM, Clark AR, Singer JA, Frame DM: Curcumin mediates both dilation and construction of peripheral arterioles via adrenergic receptors. J Invest Dermatol 131(8): 1754-1760, 2011

9. Dumont AS, Dumont RJ, Chow MM, Lin Cl, Calisaneller T, Ley KF, Kassel NF, Lee KS: Cerebral vasospasm after subarachnoid hemorrhage: Putative role of inflammation. Neurosurgery 53(1):123-135, 2003

10. Erdi FM, Guney O, Kiyici A, Esen H: The effects of alpha lipoic acid on cerebral vasospasm following experimental subarachnoid hemorhage in the rabbit. Turk Neurosurg 21(4): 527-533, 2011

11. Fassbender K, Hodapp B, Rossol S, Bertsch T, Schmeck J, Schutt S, Fritzinger M, Horn P, Vajkoczy P, Kreisel S, Brunner Z, Schmiedek P, Hennerici M: Inflammatory cytokines in subarachnoid hemorrhage. J Neurol Neurosurg Psychiat 70(4): 534-537, 2001

12. Ghaleh B, Rande J, Hittinger L, Giudicelli J, Berdaux A: Comparisons of the effects of nicorandil, pinacidil, nicardipine and nitroglycerin on coronary vessels in the conscious dog: Role of endotelium. Brit J Pharmacol 114: 496-502, 1995 
13. Gupta SC, Patchva S, Koh W, Aggrawal BB: Discovery of curcumin, a component of the golden spice, and its miraculous biological activities. Clin Exp Pharmacol Physiol 39(3): 283-299, 2012

14. Harder DR, Dernbach P, Waters A: Possible cellular mechanism for cerebral vasospasm after experimental subarachnoid hemorrhage in the dog. J Clin Invest 80: 875-880, 1987

15. Kanchan KM, Singh SK, Khosla KV, Mohindra S, Salunke $P$ : Safety and efficacy of sildenafil citrate in reversal of cerebral vasospasm: A feasibility study. Surg Neurol Int doi:10.4103/2152-7806, 2012

16. Kaura V, Bonner S: Subarachnoid haemorrhage: Early clinical indicators and biomarkers. Trend Anesthesia Crit Care 2: 4247, 2012

17. Melanie D, King BS, Jay M, Wade MF, Meiler ES, Alleyne $\mathrm{CH}$, Dhandapani MK: Curcumin attenuates hematoma size and neurological injury following intracerebral hemorrhage in mice. J Neurosurg 115(1): 116-123, 2011

18. Ozcan AT, Meral H: Transient ischemic attack after intake of sildenafil. Turk J Cerebrovasc Dis 18(1): 23-24, 2012

19. Ozdemir M, Bozkurt M, Kahilogulları G, Ugur HC, Egemen N: Subarachnoid hemorrhage and the treatment of complications. J Ankara Univ Med Faculty 64(1): 52-55, 2011

20. Ryu C, Koh J, Yu S, Kim E: Vasogenic edema of the basal ganglia after intraarterial administration of nimodipine for treatment of vasospasm. J Korean Neurosurg Soc 49:112115,2011

21. Seker F, Hesser J, Eva N, Groden G, Brocmann MA, Schubert $\mathrm{R}$, Brocmann C: Dose-response relationship of locally applied nimodipine in an ex vivo model of cerebral vasospasm. Neuroradiology 55: 71-76, 2013
22. Shimizu S, Saito M, Kinoshita Y, Ohmasa F, Dimitriadis F, Shomori K, Hayashi A, Satoh K: Nicorandil ameliorates ischemia-reperfusion injury in the rat kidney. Brit Journal Pharmocol 163: 272-282, 2011

23. Siasios I, Kapsalaki EZ, Fountas KN: Cerebral vasospasm Phrmacological treatment: An update. Neurol Res Int doi:10.1155/2013/571328, 2013

24. Soppi V, Karamanakos PN, Koivisto T, Kurki MI, Vannien $\mathrm{R}$, Jaaskelainen JE, Rinne J: A randomized outcome study of enteral versus intravenous nimodipine in 171 patients after acute aneurysmal subarachnoid hemorrhage. World Neurosurg 78(1): 101-109, 2012

25. Tapia E, Soto V, Karla MO, Guillermo Z, Eduardo M, Magdelana C, Zazueta C, Jose P: Curcumin induces Nrf2 nuclear translocation and prevents glomerular hypertension, hyperfiltration, oxidant stress, and the decrease in antioxidant enzymes in 5/6 nephrectomized rats. Oxid Med Cell Longev 2012:269039, 2012

26. Zapatero DC, Hantson P: Pharmacological treatment of delayed cerebral ischemia and vasospasm in subarachnoid hemorrhage. Ann Intensive Care 24(1): 12-20, 2011

27. Zhang L, Gu Z, Qın Z, Liang Z: Effect of curcumin on the adhesion of platelets to brain microvascular endotelial cells in vitro. Acta Pharmacol Sin 29(7): 800-807, 2008

28. Zhuang Z, Zhou M, You M, Zhu L, Ma C, Sun X, Shi J: Hydrogen-rich saline alleviates early brain injury via reducing oxidative stress and brain edema following experimental subarachnoid hemorrhage in rabbits. BMC Neuroscience 13: 47,2012 